\title{
Recensions (mai 2018)
}

Grégory Cormann \& Jérôme Englebert (dir.), Psychopathologie et philosophie. Nouveaux débats et enjeux contemporains, dans Revue Le Cercle herméneutique, vol. 26-27 (2016), 340 pages. ISBN 3412036300042. Prix : $23 €$.

Issues pour la plupart d'une journée d'étude organisée en 2015 à l'Université de Liège autour du thème : «Le philosophe dans la psychopathologie », les seize contributions recueillies dans ce numéro de la revue Le Cercle Herméneutique présentent une nouveauté parmi les publications consacrées à la promotion du dialogue entre psychopathologie et philosophie. En effet, à la différence des études de plus en plus nombreuses qui se réclament du courant anglo-américain de la «nouvelle philosophie de la psychiatrie », ce recueil n'a pas pour ambition d'établir ou de consolider les frontières d'une discipline, ni de défendre, à l'intérieur de cette discipline, un modèle méthodologique particulier. L'enjeu des études rassemblées par le psychologue Jérôme Englebert et le philosophe Grégory Cormann est avant tout d'instaurer, «avec humilité et sans ambitions démesurées » (p. 10), un espace de questionnement au croisement de disciplines et d'approches différentes et dont la finalité principale demeure de nature clinique. C'est la raison pour laquelle la relation entre philosophie et psychopathologie est envisagée à partir de la place concrète que le philosophe, avant même la philosophie, est censé occuper au sein du dispositif clinique, à partir donc d'un questionnement autour des modalités pratiques de son interaction avec les cliniciens.

En effet, comme le remarquent Christophe Adam (chap. 4 : « Le philosophe, ver du fruit et levain de la pâte ») et Giovanni Stanghellini (chap. 9: «La généalogie de la psychopathologie »), pour une large partie des psychiatres et des psychopathologues « le mot "philosophique" est synonyme de "spéculatif" » (p. 164), de sorte que la «la philosophie reste au mieux un luxueux supplément d'âme ou un vestige poussiéreux qu'on regarde avec 
condescendance » (p. 74). C'est pourquoi il faut avant tout se demander, comme le fait le psychiatre Friedrich Stiefel, pour quelle raison on devrait insérer un philosophe dans un service de psychiatrie (chap. 1: «Pourquoi un philosophe dans un service de psychiatrie de liaison?»). Autrement dit, comment, par quelles voies et avec quelles tâches le philosophe peut-il « gagner sa légitimité » (Adam, p. 68) parmi les malades et les cliniciens ?

La réponse que la plupart des contributeurs de ce recueil semble partager est que la « sagesse incompétente » (p. 10) du philosophe amènerait ceux avec qui il travaille à la "suspension volontaire des schémas habituels de faire ou de penser» (Stiefel, p. 22). Comme le remarque la philosophe Bénédicte de Villers à partir d'une expérience de terrain qu'elle a menée au Centre neuropsychiatrique Saint-Martin, dans la province belge de Namur, la rencontre avec les professionnels infirmiers et les patients autour de problématiques touchant aux dimensions pré-intentionnelles sous-jacentes aux activités de soins permettrait de «mettre en lumière ce qui se dérobe » dans ces interactions (chap. $7:$ «Vers une signification anthropologique des "dimensions informelles" des soins en psychiatrie», p. 125). Si l'on peut donc définir la psychopathologie comme une «philosophie appliquée »comme le suggèrent de manière provocatrice Englebert et Cormann en mettant l'affirmation de John Cutting en exergue dans leur introduction -, ce n'est donc pas au sens ou la philosophie aurait le rôle de «garant pour les enquêtes épistémologiques portant sur les catégories de la pensée psychopathologique» (p. 12). Tout comme la psychopathologie «n'existe pas comme domaine ou champ unifié », puisqu' «elle est traversée par des controverses qui lui donnent toute son épaisseur dynamique et heuristique » (Adam, p. 67), le philosophe non plus ne peut être dans la psychopathologie en tant que porteur d'un savoir.

C'est bien la raison pour laquelle la perspective phénoménologique apparaît comme le dénominateur commun dans la plupart des contributions présentes dans ce volume, au point que le philosophe Hubert Wykretowicz reconnaît dans cette approche la vocation naturelle de la psychiatrie (chap. 2 : «Au cœur de la psychiatrie, la phénoménologie»). Le psychiatre Thomas Fuchs, de son côté, va jusqu'à y reconnaître le fondement même (chap. 16 : «Fondements philosophiques de la psychiatrie et leur utilisation»). Si les divers modèles et méthodes phénoménologiques sont discutés et mis à l'épreuve de la clinique par certains auteurs - en particulier, Englebert et Cormann partent d'une situation clinique pour analyser le lien entre mélancolie et choc d'un point de vue qui s'inspire de la notion sartrienne de liberté (chap. 3: «Phénoménologie de l'électrochoc: une reprise du cas Jonas ») ; Gautier Dassonneville met en perspective la psychopathologie de 
l'addiction à partir de l'anthropologie de Sartre (chap. 4: «Comprendre l'addiction: réflexions à partir de l'anthropologie sartrienne »); Svetlana Sholokova se concentre sur l'événement de la rencontre entre médecin et malade à partir du « cas de Ludwig Binswanger » (chap. 13); Valérie Follet réfléchit sur les travaux de Frederik J.J. Buytendijk dans le domaine de la psychologie comparée (chap. 15: «L'homme et l'animal "en situation": commentaire sur l'approche éthologique et phénoménologique de F.J.J. Buytendijk ») - l'attitude philosophique de l'épochè est au cœur de ce volume aussi lorsqu'il n'est pas directement question de la méthode phénoménologique.

L'étude que le philosophe Antoine Janvier consacre aux expériences de Fernand Deligny avec les enfants autistes, par exemple, illustre de manière frappante ce que signifie se défaire des évidences ou suspendre le jugement quant aux enjeux et aux concepts mêmes de «thérapie », "soin », « éducation », mais aussi quant aux valeurs et aux attentes que les pratiques qui y sont liées génèrent «à l'égard de soi et des autres considérés comme sujets » (chap. 14: «Fernand Deligny ou la pensée au milieu : une généalogie du coutumier », p. 292). Un autre exemple de cette attitude critique à l'égard des «évidences perceptives construites » (p. 111) nous est offert par l'étude de la philosophe et historienne des sciences Julie MazaleigueLabaste, laquelle nous présente une analyse à la fois historique et épistémologique de la nosographie des perversions et des paraphilies afin de montrer la manière dont le travail de catégorisation psychopathologique modèle à la fois la clinique et les diagnostics (chap. 6: «Perversion, obsession, paraphilie, ou l'histoire d'un oubli : repenser la clinique des "troubles psychosexuels" "). Il s'agit là d'un cas d'étude tout à fait crucial pour montrer comment les diverses manières de concevoir théoriquement un phénomène ont des conséquences fondamentales au niveau de la clinique. Tout comme Dassonneville nous montre que penser l'addiction au cœur d'une philosophie de la liberté est ce qui va permettre au psychologue d'entamer un travail de reconstruction de l'identité narrative du patient (p.95), aussi MazaleigueLabaste souligne comment la redécouverte du rapport entre les troubles psychosexuels et les phénomènes obsessionnels détermine une prise en charge différente de ces phénomènes. De cette manière, elle met en lumière une fois de plus la «pertinence critique » que la réflexion philosophique possède pour la psychopathologie dans ses aspects théoriques comme cliniques (p. 97).

Par rapport à la première partie, la seconde partie du volume, consacrée à «La philosophie dans la psychopathologie », présente des recherches qui recoupent certaines des discussions les plus actuelles et fécondes dans le 
domaine de la «philosophie de la psychiatrie ». Le thème du caractère préintentionnel ou préréflexif de la présence au monde, en particulier, est un sujet qui, selon de divers angles d'analyse, est commun aux contributions de Stanghellini (chap. 9), Louis A. Sass (chap. 10: «Les paradoxes de la réflexivité »), Jérôme Englebert et Caroline Valentiny (chap. 11 : «Le schizophrène comme hyper-philosophe ») et Michel Dupuis (chap. 12: «Significativité et Daseinsanalyse »). L'un des aspects à notre avis les plus intéressants de cette problématique est la manière dont la recherche sur le sens commun et la constitution de l'expérience croisent des thèmes et des méthodes relevant des domaines de l'ethnographie et de l'anthropologie culturelle. Stanghellini, par exemple, affronte le problème de la compréhension intersubjective en se référant aux études ethnographiques d'Ernesto De Martino et il suggère d'appliquer le modèle de l' « ethnocentrisme critique » au domaine de la psychopathologie (p. 174). C'est dans ce même esprit que se situe également l'étude historique et systématique de Michel Dupuis sur le statut de la «significativité». Cette recherche se propose en effet de considérer aussi les dimensions de l'espace-temps culturel dans la perspective phénoménologique de l'« évidence naturelle ». La significativité, précise Dupuis, est «constituée par une forme de communauté »(p. 242), d'où la nécessité pour le psychiatre de faire preuve d'une véritable « lucidité anthropologique » qui lui permette de dépasser le cadre individuel de son analyse de cas cliniques (p. 241).

Il s'agit là sans doute de l'un des enjeux les plus intéressants de ce recueil et qu'il vaudrait surement la peine d'approfondir dans le futur. $\mathrm{Si}$, en effet, l'anthropologie philosophique qui a accompagné historiquement l'approche phénoménologique de la psychopathologie semble de plus en plus disparaître aujourd'hui au profit d'un programme «néo-phénoménologique » rallié aux neurosciences, on assiste ici, en revanche, à une sorte de réactualisation de la réflexion anthropologique qui prend comme base une approche «pragmatique » de l' « être dans le monde ». Or quelle est la nature de ce nouveau rapprochement entre philosophie, anthropologie et psychiatrie? S'agit-il d'une simple métaphore méthodologique ou bien d'un véritable croisement de savoirs qui pourrait donner lieu à une nouvelle forme d'« anthropologie philosophique »?

Elisabetta Basso 
Lucia Angelino (dir.), Quand le geste fait sens, préface de Renaud Barbaras, Milan, Mimesis, 2015, 206 pages. ISBN 9788869760167. Prix : 20,00€.

Quand le geste fait sens paraît à un moment où le concept de geste fait florès dans les sciences humaines : depuis la sémiologie phénoménologique des Gestes de Vilém Flusser (série d'essais parus de 1948 à 1990 et réunie chez Al-Dante en 2014 ) à la philosophie des gestes ordinaires de Barbara Formis $^{2}$, des Gestes d'humanité d'Yves Citton ${ }^{3}$ à la pensée du Geste mineur chez Erin Manning ${ }^{4}$, une "gestologie» semble se développer depuis la manière incarnée, concrète et écologique dont les échanges se trament par le mouvement entre les humains, leurs congénères et leurs milieux.

La question où Quand le geste fait sens aborde les gestes est celle, comme le titre l'indique, de la signification. Mais les auteurs ne s'intéressent pas tant au geste comme porteur de signification - comme si un vécu ou une pensée verbale devaient être traduits par des gestes - que comme moyen par lequel le sens se fabrique - ce que l'anglicisme «faire sens » veut pointer. On reconnaît là une compréhension phénoménologique de l'expression: le sens n'est pas ce qui serait tenu en une réserve privée qui viendrait ensuite à être transmis dans l'extériorité comme on fait passer un liquide d'un récipient à l'autre ; le sens est immanent à ses modes d'expression. Mais alors que la phénoménologie s'est habituellement cantonnée à penser cette venue-au-sens dans la langue parlée ou écrite, le propos des auteurs de Quand le geste fait sens est de faire place à d'autres gestes que ceux qui mouvementent la bouche de celui qui parle ou la main de celui qui écrit : des gestes de danse, des gestes de musique, des gestes de dessin, des gestes de performance, des gestes de la vie, et des gestes, même, de philosopher ${ }^{5}$.

${ }^{1}$ Vilém Flusser, Les gestes, texte établi par Marc Partouche, Marseille, Al DanteAka, 2014.

2 Barbara Formis (éd.), Gestes à l'œuvre, Paris, De l'incidence, 2008 ; Barbara Formis, Esthétique de la vie ordinaire, Paris, PUF, 2010.

3 Yves Citton, Gestes d'humanités. Anthropologie sauvage de nos expériences esthétiques, Paris, Armand Colin, 2012.

${ }^{4}$ Erin Manning, The Minor Gesture, Durham, Duke University Press, 2016.

${ }^{5}$ Citons, parce que nous n'aurons pas l'occasion de revenir à eux dans l'espace de cet article, les articles qui élargissent le propos à ces autres gestes de création : celui de Cécile Angelini, «Traces conceptuelles » (p. 129-147), consacré aux gestes de tracer notamment dans l'action painting; celui de Richard Shusterman, «Le philosophe sans la parole » (p. 149-166) consacré au philosopher considéré comme exercice spirituel et esthétique ; celui de Barbara Formis, «La performance de la vie»(p. 167-184), consacré aux gestes ordinaires; et celui de Jessica Wiskus,

\section{5}

Bull. anal. phén. XIV 4 (2018)

https://popups.uliege.be/1782-2041/ (c) 2018 ULiège BAP 
Dans cette étude critique, nous proposons de resituer les interrogations de cet ouvrage dans une brève histoire des concepts de geste et de mouvement, à la suite de quoi nous proposons une perspective critique sur deux concepts transversaux dans l'ouvrage : ceux de «geste intérieur » et de « geste partagé ».

\section{Gestus et modernité}

Quand Cicéron et Quintilien introduisent le concept de gestus dans la langue latine $^{1}$, c'est dans le contexte de leurs traités de rhétorique (le De Oratore pour le premier, l'Institution oratoire pour le second) où ils reprennent le thème cher aux rhéteurs grecs de la sémiotique corporelle qui soutient le discours : avec la parole, la soutenant, il y a une manière de se tenir, une posture, certains mouvements des mains, du tronc et du visage qui contribuent à la fabrique du discours prononcé face à une assemblée. Sous le mot de geste, au moment même de son apparition, c'est donc d'abord cette idée de signifiance des mouvements et des postures humaines qui est pointée.

Les rhéteurs étaient en effet très conscients de la manière qu'ont les gestes de déterminer le sens d'un discours et c'est généralement avec une certaine méfiance qu'ils considéraient la puissance signifiante du geste. Ainsi Pythagore pour faire cours se cachait-il derrière un rideau afin que ses auditeurs non-initiés puissent suivre le déploiement de ses arguments sans être sous la dépendance des gesticulations qu'il pouvait être tenté de faire venir au secours de ses raisonnements. Inversement, Platon, conscient du lien puissant entre geste et disposition à la pensée préconise au philosophe-roi de faire un usage politique des arts du geste que sont la danse et la musique : dans les Lois, il propose d'apprêter les citoyens à certaines idées en les faisant bouger selon certaines mesures ou démesures, seuls ou ensemble,

«Temporalité et rythme musical dans Les confessions de Saint Augustin » (p. 185197), consacré au geste d'écrire et de méditer la philosophie tel qu'il est pratiqué par Augustin.

${ }^{1}$ Gestus, participe passé de gero («porter» ou «se porter»), traduit la périphrase grecque schèmata tou somatos, c'est-à-dire « la figure du corps », « la disposition », « l'attitude du corps » $c f$. sur ce point l'entrée « Gestus » du Brill's New Pauly; en ligne, referenceworks.brillonline.com/browse/brill-s-new-pauly, consulté le 10 octobre 2017. 
pour fixer ainsi à leurs articulations des manières d'être et de percevoir le monde ${ }^{1}$.

Pour une variété de raisons et selon les lignes d'une histoire complexe qu'ont notamment retracée Jean-Claude Schmitt pour le Moyen-Âge ${ }^{2}$ et Adam Kendon pour la Modernité ${ }^{3}$, ce lien fondamental entre le geste et le sens en est venu à s'effriter au point d'arriver, à l'orée du XX $X^{\mathrm{e}}$ siècle, à leur pure et simple dissociation dans la linguistique structurale saussurienne, où le geste est expulsé hors du domaine de l'étude de la communication humaine. Comment Saussure, en effet, figure-t-il l'échange linguistique ? Tantôt comme deux têtes suspendues dans le vide ${ }^{4}$, tantôt plus classiquement encore comme les lignes d'un dialogue sans didascalie où poliment, $\mathrm{A}$ et $\mathrm{B}$ s'échangent des mots sans qu'aucun autre signe ne vienne perturber la transmission de l'information.

Bien que les divers courants de la paralinguistique ${ }^{5}$ nous aient appris depuis à réintégrer les gestes à l'ordre de la production signifiante chez les humains, une question est restée et reste en suspens : celle de savoir ce qui a autorisé l'expulsion moderne du sens hors du corps des parlants pour ne le faire résider que dans les mots qu'ils se disent, à eux-mêmes ou aux autres.

La préface de Renaud Barbaras à Quand le geste fait sens (p. 9-14), offre un début de réponse à cette question en examinant l'une des décisions théoriques majeures de la modernité scientifique: la séparation de deux

\footnotetext{
${ }^{1}$ C'est ce que Philippe Lacoue-Labarthe a appelé, dans un essai sur la « Typographie » (Mimèsis des articulations, Paris, Aubier, 1975), « l'ontotypologie » platonicienne.

2 Jean-Claude Schmitt, La Raison des gestes dans l'Occident médiéval, Paris, Gallimard, 1990 ; Les Rythmes au Moyen Âge, Paris, Gallimard, 2016.

3 Adam Kendon, Gesture. Visible Action as Utterance, New York (NY), Oxford University Press, 2004.

${ }^{4}$ Cf. Ferdinand de Saussure, Cours de linguistique générale (1916), Paris, Payot, 1995, p. 27.

${ }^{5}$ Dans la lignée des travaux novateurs de Ray Birdwhistell (Kinesics and Context. Essays on Body Motion Communication, 1970) et d'Edward T. Hall (La dimension cachée, 1966). Ces nouvelles disciplines ont entraîné dans leur sillage des effets de fascination béate et des récupérations managériales par des auteurs peu scrupuleux, cherchant à transformer ces analyses en catalogues de formules à appliquer pour « décoder » et manipuler les comportements d'autrui. Mais ces dérives et les similisavoirs qui y sont attachés sont bien loin de la rigueur descriptive des fondateurs de la para-linguistique. $C f$. à ce sujet l'article à charge de Pascal Lardellier, «Pour en finir avec la "synergologie" », Communication, vol. 26/2, 2008.
} 
domaines d'investigation jusqu'alors entremêlés, celui de la matière et celui de l'esprit.

En quoi cette séparation concerne-telle le lien (ou l'absence de lien) du geste à la production du sens ? Elle le concerne dans la mesure où cette séparation des domaines matériel et spirituel, qui est l'acte de fondation moderne de la science physique (Galilée) et de la métaphysique (Descartes), repose sur une opposition plus profonde, sur un dualisme plus originel que celui qui sépare l'âme et le corps : il s'agit de l'opposition qui sépare l'ordre du sens comme ordre du vécu (objet de l'enquête métaphysique et psychologique) et de l'ordre du mouvement comme ordre de l'étendu où rien n'arrive véritablement que des modifications de lieu (objet de l'enquête physique et mathématique).

On peut s'en assurer en contrastant (à la manière de Jan Patočka dans Aristote, ses devanciers, ses successeurs) la science moderne avec la physique aristotélicienne. Comme le synthétise Renaud Barbaras, en effet,

dans la perspective aristotélicienne, le mouvement renvoie à une faiblesse ontologique, à un défaut d'être et doit donc être compris comme le procès par lequel la substance rejoint son être, devient ce qu'elle est : c'est un mouvement d'accomplissement qui possède une signification ontologique (p. 9).

Or que se passe-t-il avec la mathesis universalis ? L'inachèvement disparaît, le monde devient pleinement actuel, pleinement réalisé. Mais évidemment, le mouvement (le phénomène du mouvement) n'a pas disparu : simplement, ce mouvement ne peut plus être compris comme accomplissement, puisque tout est donné, puisque le monde est «plat», sans puissance. Ainsi que le dit à nouveau Renaud Barbaras, dans le monde de la physique moderne,

l'étant matériel ne contient aucune réserve, aucune puissance, aucune profondeur ontologique; il n'est que ce qu'il est, tout entier déplié, extérieur à luimême ; il est l'extériorité comme être, c'est-à-dire étendue. De cette étendue, le mouvement ne peut alors être qu'un mode, et à ce titre, il se confond avec le mouvement local : il est un simple état, qui, à l'instar de l'immobilité, persiste tant qu'une force ne vient le contrarier. Autant dire qu'un tel mouvement, indifférent à l'essence de l'étant qui est en mouvement, n'a plus aucune valeur ontologique : il ne fait rien, n'accomplit rien, et ne va donc nulle part. Il n'a aucune direction ontologique, c'est-à-dire aucun sens ; il est aussi étranger au sens que l'étendue l'est à la sphère de la pensée (p. 9-10).

Telle est la leçon conjointe de Galilée et de Descartes : là où il y a du sens, il n'y a pas de calcul possible (l'âme n'est pas mathématisable), mais cette 
impossibilité est un prix peu cher payé pour le gain immense qu'il autorise par ailleurs, à savoir que là où il n'y a pas de sens, tout (en droit) peut être algébrisé.

Ce «tout», dont le livre est écrit en langage mathématique, devient par conséquent impensable dans la langue naturelle. Galilée l'appelle encore du nom de «nature », mais c'est un nom usurpé puisque c'est une nature où plus rien n'est natus, où plus rien n'est né, et où tout est étendu. La nature physique devient ainsi étrangère au sens, qui quant à lui se mêle et se pense inévitablement dans une parole incarnée ${ }^{1}$.

Comment, depuis cette Modernité qui nous échoit, faire place à des mouvements qui ne seraient pas étrangers à l'ordre du sens, mais qui au contraire exhiberaient de part en part une signifiance inséparable de la motricité ? C'est à fourbir les armes pour répondre à cette question que les différents essais réunis dans le livre de Lucia Angelino sont dédiés. Ils le font en s'intéressant à une catégorie de mouvements bien particulière, à savoir les gestes impliqués dans la création artistique. En effet,

qu'il s'agisse de la danse ou des autres arts, on y fait l'épreuve d'un sens qui s'avère irréductible à l'idée, qui est de part en part incarné, c'est-à-dire inséparable de son effectuation ou de sa profération, d'un sens qui n'est signifiant qu'à la condition d'être toujours moins que lui-même, d'être sa propre imminence (p. 11).

Les gestes de l'art, et même plus généralement les gestes de la création (y compris philosophique), attestent ainsi d'une pensée qui ne se tient pas dans un espace anti-gestuel, mais tantôt au bord des gestes qui l'exprime (en quoi le sens est «moins que lui-même » et «sa propre imminence ») tantôt à même ces gestes (en quoi il est « inséparable de son effectuation»).

Dans les pages qui suivent, nous voulons détailler deux aspects du geste tels qu'ils sont pensés dans l'ouvrage : le «geste intérieur » d'une part et le « geste partagé » d'autre part, dont nous verrons qu'ils permettent de penser ce double statut du sens comme imminent («moins que lui-même ») et comme immanent («inséparable de son effectuation») aux gestes qui l'expriment.

${ }^{1}$ C'est la séparation latine du logos des Grecs en ratio (raison, calcul) d'un côté et lingua (langue, parole) de l'autre. 


\section{Le geste intérieur}

Les gestes ne sont pas seulement des mouvements que nous faisons dans le monde et avec les autres, ce sont des mouvements que nous nous sentons faire et même, plus avant, avec lesquels nous sentons.

En français, des cinq sens classiques, seul le «toucher» (à la fois substantif et verbe) semble retenir cette idée que pour sentir il ne suffit pas de recevoir : il faut encore bouger (palper, glisser, gratter). Mais à dire vrai, tous les autres systèmes perceptifs impliquent un «bouger » (c'est-à-dire un bouger qui est indissociablement un être-bougé) similaire: un tendre l'oreille, un savourer, un humer, un zyeuter. Comme l'a répété à l'envi le psycho-phénoménologue Erwin Straus dans son traité $\mathrm{Du}$ sens des sens, sentir et se mouvoir sont les deux faces d'une même pièce. Pour une philosophie des gestes, cette maxime doit s'entendre comme la corrélation permanente entre l'épreuve du monde et l'attitude ou la disposition corporelle qui la soutient - ce qu'on appellerait en toute rigueur lexicale, une «posture », au sens à la fois littéral et figuré, comme quand on parle d'une « posture d'écoute » ou d'une « posture de refus».

Ce point de vue sur le vécu du geste n'est donc pas ou pas seulement un point de vue sur l'expérience interne, «musculaire ou neuronale » (p. 56) comme dit Lucia Angelino reprochant à certaines approches (en esthétique et dans les sciences empiriques notamment) de se cantonner aux seules kinesthésies dans leur étude du mouvement. C'est plutôt un point de vue phénoménologique qui, «dans le sillage ouvert par Erwin Straus et Maurice Merleau-Ponty » (idem), veut intégrer et renommer les qualités dynamiques ou rythmiques d'une expérience qui est, quant à elle, inséparablement une expérience du monde et de soi.

C'est Frédéric Pouillaude, dans l'article qu'il consacre à la question de «L'expression en danse » (p. 35-50), qui détaille avec le plus de force et de précision les diverses qualités dynamiques à l'œuvre dans les gestes. Restituons cette approche : elle nous paraît paradigmatique des bénéfices que cet ouvrage sait fournir à qui veut penser en gestes ${ }^{1}$.

C'est dans le système Effort élaboré par le danseur et chorégraphe Rudolf Laban à partir des années 1940 que Frédéric Pouillaude trouve les

1 Les articles de Lucia Angelino, «Le mouvement vécu dans l'improvisation musicale » (p. 53-72) et de Jean-Marc Chouvel, «Le geste intérieur du musicien » (p. 73-90) fournissent un appui supplémentaire à cette analyse, eux qui détaillent la manière dont le geste musicien n'a pas seulement vocation à produire des résultats sonores mais est «un geste vital qui sonne comme forme de l'être au monde » (p. 80). 
linéaments d'une théorie des gestes. Ce système permet singulièrement de répondre à une difficulté que rencontre Pouillaude en tant que philosophe de l'art s'inscrivant dans la tradition goodmanienne (qui parle de langages de l'art) : si la danse est structurée comme un langage, de quoi ce langage est-il le nom ? Et plus spécifiquement, comment peut-il y avoir un langage où quelque chose serait communiqué entre deux régimes d'expérience radicalement asymétriques : celui du danseur, dont l'expérience en première personne est majoritairement kinesthésique; et celui du spectateur dont l'expérience en troisième personne est majoritairement visuelle ? Comme Frédéric Pouillaude le souligne,

toute théorie du spectacle de danse doit à un moment ou à un autre s'affronter à cette asymétrie sensorielle fondamentale et supposer une intermodalité où le visuel et le kinesthésique puissent réciproquement s'échanger. La notion d'empathie est le nom classique donné à une telle intermodalité (p. 44).

Mais comme le philosophe le pointe, cette notion n'est pas une solution elle est simplement le nom du problème qu'il s'agit de résoudre, à savoir : qu'est-ce donc qui circule ainsi d'une modalité sensorielle à l'autre ? La réponse que fournit (incidemment, sans spécifiquement chercher à résoudre cette question) le système Effort de Laban consiste dans la combinatoire descriptive qu'il fournit pour rendre compte du mouvement. Pour Laban, en effet,

tout mouvement humain est investi d'un effort spécifique, et cet effort se décompose en différentes attitudes à l'égard des principaux facteurs du mouvement que sont l'espace, le temps, le poids et le flux. Pour simplifier l'analyse, on assigne à chaque facteur deux valeurs et deux seulement : le rapport à l'espace pourra être soit direct, soit flexible ; le rapport au temps soit soutenu (continu), soit soudain ; le rapport au poids soit ferme, soit léger; le rapport au flux soit contrôlé, soit libre (p. 47).

À partir de ces coordonnées, Laban déduit, par combinaison, une liste de gestes archétypaux qui permettent de spécifier, selon lui, les qualités gestuelles fondamentales de tout mouvement. Le geste de presser par exemple qualifie tout mouvement qui entretient un rapport à l'espace direct, un rapport au temps soutenu, et un rapport au poids ferme. Le geste de glisser, son plus proche parent, entretient un rapport à l'espace également direct, un rapport au temps également soutenu, mais son rapport au poids sera dit léger. Et ainsi de suite avec les verbes/gestes de frapper, de tapoter, de tordre, de flotter, de fouetter et d'épousseter, tous gestes qui concernent 
d'abord des gestes de la main mais qui doivent être étendus au style général d'un mouvement et à la posture qui les soutient ${ }^{1}$.

En quoi ces qualités dynamiques que nomment les styles d'efforts nous permettent-elles de rendre compte de la part à la fois sensible et visible du geste ? Elles le permettent en ce qu'elles décrivent une attitude intérieure qui «n'est pas un mystérieux dedans dérobé au regard du spectateur» mais qui « est aussi bien un strict contenu de visibilité, un dedans immédiatement exposé »(p. 49). Les efforts, pour le dire autrement, ont une existence intermodale ou amodale : ils existent entre ou plutôt en-deçà de la distinction des sens entre eux - ils sont la part apparaissante de mon intériorité, aux autres et à moi-même.

Frédéric Pouillaude, à l'appui de Laban, donne ainsi les moyens de penser un mode d'existence alternatif à la signification dénotative de gestes. Pour signifier, les gestes n'ont pas besoin de pointer vers un sens qu'ils ne contiendraient pas (et cela ne les condamne pas à "se signifier eux-mêmes » comme on a pu le dire de certaines danses post-modernes comme celles du Judson Church Theater occupées à sursignifier leur propre «danséité » en disant : «voilà ce que je vous montre : un corps qui danse »), elles peuvent au contraire pointer vers une partie d'elles-mêmes, une intériorité du geste qui n'est pas une intériorité d'idées, mais une intériorité tissée de vécus dynamiques.

\section{Le geste partagé}

La question du mode d'existence intersubjectif des gestes, qui trouve une réponse partielle dans le concept de «geste intérieur» comme qualité dynamique à la fois ressentie et sensible à d'autres, n'est toutefois pas entièrement réglée.

C'est ce qui apparaît au chapitre « Sur la musique faite ensemble » (p. 91-109), où Laurent Perreau déplace la question de la signifiance intersubjective des gestes hors des sentiers battus de la relation entre

\footnotetext{
${ }^{1}$ Le fait que les gestes de la main soient les gestes élus par Laban pour paradigmes de la motricité humaine en général pourrait faire penser à une dette du chorégraphe à l'égard des chirologies des rhéteurs antiques. C'est sans doute partiellement le cas, comme l'attestent les nombreuses références de Laban au mime et au comédien. Mais on peut aussi expliquer cette référence phénoménologiquement, par le fait que les mouvements de la main, probablement en raison de la finesse sensori-motrice, ont été l'occasion d'une prolifération lexicale sans pareil dans les langues vernaculaires : il n'y a guère que pour les mains (et pour les animaux) que nous ayons des verbes de mouvement précis et imagés.
} 
performeur et spectateur pour se poser la question des relations des agents entre eux. Le problème n'est plus alors celui de la dissymétrie entre le faire (du performeur) et le sentir (du spectateur) mais plutôt celui d'un type singulier d'intentionnalité : "l'intentionnalité collective». Ce syntagme forgé par John Searle ${ }^{1}$ indique une spécificité de l'expérience subjective à l'intérieur de certaines actions partagées où s'observe « une communauté d'expérience qui ne se laisse pas réduire à une somme ou une collective d'intentions individuelles, ainsi que la conscience partagée d'une implication commune des acteurs dans l'action» (p.92). L'intentionnalité collective nomme ainsi le fait que dans certaines activités faites ensemble (comme la musique, la danse, l'amour...), il y a un «engagement conjoint dans l'action » (idem), c'est-à-dire une forme de partenariat: la reconnaissance d'une participation active de chacune des parties impliquées dans l'activité.

Le geste paradigmatique employé pour penser l'intentionnalité collective est, depuis un article célèbre de Margaret Gilbert, le geste de marcher ${ }^{2}$. Qu'est-ce qui permet de dire que deux personnes marchent ensemble? L'article de Gilbert envisage une femme, Sue Jones, qui marche le long d'une route: un homme la rejoint et marche, durant quelques secondes ou pendant un certain temps, au même rythme qu'elle, apparemment dans la même direction qu'elle, à une distance qui, en raison de l'étroitesse du trottoir, pourrait faire penser à un observateur extérieur qui viendrait d'arriver, qu'ils se promènent effectivement ensemble, et que leurs chemins ne se sont pas seulement rejoints de manière accidentelle. Que suppose l'observateur extérieur dans un tel cas ? Il suppose, argumente Gilbert, que les deux acteurs se sont donnés un but commun qui dépasse leurs individualités, c'est-à-dire qu'ils marchent au «nous » plutôt qu'au $\ll \mathrm{je} »$ :

Marcher ensemble avec une autre personne implique de participer à une activité d'un type spécial, activité dont le but est entretenu par un sujet pluriel, par opposition à une activité dont le but serait seulement partagé par différentes personnes. [...] Je suppose qu'il y a de nombreuses activités de cette sorte, et qu'on peut les désigner comme des actions "partagées", «conjointes » ou «collectives». Des exemples de ces activités incluent

\footnotetext{
${ }^{1}$ John Searle, «Collective Intentions and Actions », in P. Cohen, J. Morgan, and M.E. Pollack (éds.), Intentions in Communication, Cambridge (MA), MIT Press, 1990.

2 Margaret Gilbert, «Walking Together. A paradigmatic social phenomenon», Midwest Studies in Philosophy, vol. 15, 1990.
} 
voyager ensemble, manger ensemble, danser ensemble, élucider un meurtre ensemble, et ainsi de suite ${ }^{1}$.

La proposition de Laurent Perreau est d'élargir la compréhension de ce phénomène au geste de faire de la musique ensemble. Il s'appuie pour ce faire sur une formule remarquable proposée par le phénoménologue Alfred Schütz pour décrire la relation entre deux personnes (deux lutteurs, deux escrimeurs, etc., mais aussi bien deux personnes qui font l'amour, qui marchent ensemble, qui dansent ensemble) engagées dans une activité commune : l'idée qu'entre ces deux personnes, une « relation d'accordage mutuel » (mutual tuning-in relationship) se déploie ${ }^{2}$.

Dans la traduction française de l'article, le mot de «syntonie » a été choisi pour remplacer cette périphrase, et on ne peut que le regretter : s'y perdent les idées de mutualité (à peine sensible dans le syn- de «syntonie ») et de processus (tout bonnement effacé par le substantif en français, alors que l'anglais utilise un verbe au gérondif, c'est-à-dire désigne un accordage, tuning, et non un accord). Or que dit cette formule profonde de Schütz ? Elle indique que l'accordage n'est pas un «pré-requis » qui permet le bougerensemble, mais qu'au contraire, le bouger-ensemble est lui-même une relation d'accordage mutuel. Autrement dit, nous ne bougeons pas ensemble parce que nous nous y sommes accordés : au contraire, on peut dire que nous bougeons ensemble dans la mesure où justement nous ne présupposons pas l'accord mutuel, mais cherchons à nous ajuster l'un à l'autre. Autrement dit, bouger ensemble ne suppose pas l'accordage: bouger-ensemble c'est s'accorder.

Alfred Schütz dit dans ce sens que la syntonie se situe en-deçà ou « au fond de toute communication possible $»^{3}$. Et il faut bien comprendre pourquoi : non pas parce que rien n'y est communiqué, ou parce qu'il y aurait une telle fusion des individualités que l'expérience partagée n'aurait plus à être exprimée, mais parce que la relation dont il s'agit ne se fait pas en fonction d'un langage (code ou langue). Le partage se fait dans l'invention, ou du moins dans le désir d'inventer, justement d'une manière propre à cette relation, l'agir ensemble. On objectera à raison qu'en musique comme ailleurs, une telle improvisation pure n'existe pas, qu'il n'y a pas de partage

${ }^{1}$ Ibid., p. 9 (notre traduction).

${ }^{2}$ Alfred Schütz, «Faire de la musique ensemble. Une étude des rapports sociaux », traduit de l'anglais (États-Unis) par l'équipe éditoriale de la revue, Sociétés, 2006/3 (numéro 93), p. 15-28.

${ }^{3}$ Ibid., p. 25. 
gestuel qui ne se fasse sur fond d'une certaine limitation des possibilités d'action. Mais l'idée que bouger-ensemble est une relation d'accordage mutuel ne suppose pas l'éradication de tout code : elle indique simplement que l'enjeu de la relation n'est pas la communication d'informations par le biais d'un système de signes préétablis - qu'au contraire il est ou se veut la création commune et momentanée d'un tel système ou du moins d'un soussystème «à deux » qui n'obéisse pas seulement aux lois de la langue, mais aussi aux règles que nous nous y donnons le temps de notre rencontre.

Laurent Perreau parle ainsi d'un " "sens" natif, d'ordre prélinguistique et précommunicationnel, se développant dans l'interaction des gestes, des mouvements corporels et des actions » (p. 106). C'est de ce sens émergeant qu'il est question dans le bouger-ensemble, et spécifiquement dans la musique faite ensemble.

Les réflexions d'Anne Boissière sur «Le pouvoir du jeu» (p. 113127) complètent et renforcent cette analyse de l'intentionnalité collective en l'élargissant à d'autres êtres que les partenaires humains. L'analyse de Laurent Perreau se situe en effet résolument dans la filiation de la phénoménologie des actes sociaux, et reste donc pour l'essentiel cantonnée aux gestes que les humains s'adressent entre eux. Le geste de jouer (principalement tel qu'il est pratiqué par les enfants) où Anne Boissière situe sa réflexion excède cette socialité et intègre, comme partenaires de gestes conjoints, diverses entités extra-humaines : les jouets, les éléments, la nature, avec lesquels l'enfant au jeu est impliqué dans une relation qui imite (si elle ne la fonde pas) l'intentionnalité collective qu'on trouve dans les gestes (de danser, de musiquer, etc.) faits ensemble avec d'autres humains.

Le point de départ de la réflexion d'Anne Boissière est un aspect phénoménologique crucial de l'intentionnalité collective, à savoir le sentiment/l'expérience, pour les agents engagés dans une action collective, d'être saisis, emportés par un geste, un mouvement, un faire plus larges qu'eux (ce que Margaret Gilbert appelait le passage d'une intentionnalité centrée sur le moi à une intentionnalité centrée sur le nous). Pour Anne Boissière, cet être-saisi est une caractéristique fondamentale de l'attitude ludique où, comme elle le dit en citant Buytendijk, « on ne joue jamais seul, quand bien même le partenaire serait invisible ; on ne joue jamais qu'avec un objet qui à sa façon joue avec le joueur », c'est-à-dire que «l'objet ne participe au jeu que s'il peut, dans son mouvement propre ou son élasticité, se prêter à une logique de l'apparition saisissante » (p. 122). Ainsi les jouets sont-ils élus pour leur capacité à non seulement me répondre, mais encore à me commander, à me solliciter en me proposant de m'accorder avec eux comme je m'accorde avec des partenaires humains dans la musique, la danse 
ou toute autre action collective en général réglée par le plaisir de bouger ensemble.

Le texte d'Anne Boissière est frappant pour ce qu'il suggère d'homologie entre l'action collective et le jeu solitaire de l'enfant qui, en matière de partenaires avec qui consoner, se donne - pour ainsi dire - le monde luimême. Ce monde n'est cependant pas le même que le monde de l'adulte avec ses pôles et ses significations arrêtées : «L'enfant n'a pas affaire à des objets fixes, mais à des esquisses de formes, à un monde mouvant, au sein duquel des formes saisissantes apparaissent; il se laisse happer ou saisir par ses formes» (p. 124). Le jeu prend ainsi au sérieux l'idée que la vie est Auseinandersetzung : "conversation», «débat» avec le milieu. Il fait du monde et de ses objets des comportements à peine esquissés et qu'il s'agit de deviner, avec lesquels il faut négocier. Il insère donc la «relation d'accordage mutuel »(mutual tuning-in relationship) dans les interstices de la distance qui sépare ordinairement les sujets des objets : il instaure un geste partagé, non seulement avec d'autres humains, mais encore avec les autres non-humains qui nous entourent.

Le jeu est ainsi, selon l'argument d'Anne Boissière, l'occasion de transformer les Gestalten, les formes arrêtées de notre monde environnant, en Gestaltungen, en formations. On en trouve modèle dans l'appréhension enfantine des mouvements animaux :

Pour l'enfant, ce n'est pas l'objet « poisson » (sous-entendu « qui nage ») qui importe, relation uniquement pertinente dans une logique du savoir qui n'est pas la sienne; l'enfant compte avec le mouvement uniquement, et dans la mesure où celui-ci rend possible l'émergence des formes, elles-mêmes vivantes et éphémères : c'est dans la nage en mouvement, et dans un mouvement qui est aussi celui de la rivière, que la forme du poisson, ou plus exactement l'esquisse d'une forme de poisson se présente (p. 1241).

\footnotetext{
${ }^{1}$ L'expression « la nage poissonne » (qu'Anne Boissière trouve chez Henri Maldiney) vient probablement de Jean Paulhan, que cite Sartre dans le catalogue d'une exposition du peintre Paul Ribeyrolles : «Une toile ne parle pas — ou si peu. Quand elle discourt, le peintre fait de la littérature. Rebeyrolle n'en fait jamais ; quand je regarde ses rivières et ses truites, je me rappelle ce mot d'un philosophe chinois, inventé par Paulhan : "la nage poissonne". Pourtant voici la seconde série d'œuvres engagées qu'il nous donne en deux ans. S'engager, n'est-ce point dire ? Et quand, pour parler comme Tardieu "le vert hésite à peindre des feuilles", pourquoi le rouge accepterait-il de peindre du sang ? » (cité par Alain Leduc dans « Paul Ribeyrolle. Le paysage d'une perdrix », La Revue commune, \#38, juin 2005). Une idée similaire est exprimée par le philosophe dans un article inédit sur André Masson : «Voilà ce que
} 
On retrouve ainsi, à nouveau, cette idée d'un sens émergent de notre rapport au monde ou aux autres, plutôt qu'un sens pré-donné et qu'il faudrait simplement communiquer. Le parcours des gestes de jouer de la musique ou de jouer (tout court) permet de mettre au jour quelles dispositions sont requises pour qu'un tel sens puisse émerger de nos gestes au lieu de leur préexister : il s'agit d'être disposé à reléguer une certaine centration subjective, d'accepter d'être momentanément emporté par, de partager, un temps, un geste avec un autre être.

\section{Conclusion}

Quand le geste fait sens donne ainsi de nombreux coups de sondes dans un espace théorique qui reste encore à peine exploré par la phénoménologie et par la philosophie en général, espace que le philosophe Jean Clam a circonscrit dans ses plus récents travaux comme espace de «l'infra-motilité » ${ }^{1}$. Cet espace - qui pourtant intéresse la phénoménologie de l'expression au premier chef, puisque c'est l'espace où le sens s'esquisse, se trame sans s'être encore donné - gagne à être considéré du point de vue des gestes de création pour deux raisons au moins.

La première est qu'en ces gestes, se dessine avec le plus de clarté l'indistinction du sentir et du se mouvoir - le geste créateur est «mouvementement », selon un autre néologisme forgé par Jean $\mathrm{Clam}^{2}$ : inséparablement bouger et être-bougé ${ }^{3}$.

La seconde est que le geste échappe à la distinction entre sujets humains et sujets non-humains : alors que le sens qui se prépare dans l'infralangue garde inévitablement quelques traces d'autrui (le pré-verbal suppose bien qu'à un moment, il y aura quelque chose à dire à quelqu'un), le sens qui

Masson veut peindre à présent : ni l'envol, ni le faisan, ni l'envol du faisan : un envol qui devient faisan ; il passe dans le champ, une fusée éclate dans les buissons, éclatefaisan : voilà son tableau. » (cité par Bernard Vouilloux dans « Le texte et l'image », Littérature, vol. 87(3), 1992, p. 98).

${ }^{1}$ Cf. notamment Jean Clam, Genèses du corps : des corps premiers aux corps contemporains. Une théorie des mouvements corporants, Paris, Ganse Arts et Lettres, 2014 , p. 50 et suivantes.

2 Jean Clam, Orexis, désir, poursuite. Une théorie de la désirance, Paris, Ganse Arts et Lettres, 2012.

${ }^{3}$ Comme nous l'avons dit plus haut, certains mouvements que nous faisons sont tout autant des mouvements qui nous font : ce sont des mouvements qui ont leur propre movimentum, leur propre élan ou allure (ce que l'anglais, par crase, appelle leur momentum). Lucia Angelino le dit en passant dans une formule saisissante à propos du geste improvisé : c'est « la merveille d'être prédisposé par ce qu'on fait » (p. 66).

17

Bull. anal. phén. XIV 4 (2018)

https://popups.uliege.be/1782-2041/ @ 2018 ULiège BAP 
se prépare dans l'infra-geste fait la place à d'autres entités qu'humaines qui peuvent ainsi circuler et contribuer à la genèse du sens.

Les articles de Quand le geste fait sens que nous avons mentionnés touchent à ce lieu où le sens se fabrique. Ils le font en donnant des éléments descriptifs et théoriques pour penser la signifiance propre aux formes dynamiques des gestes, et s'appuient, avec ingéniosité, sur des écrits d'artistes, des témoignages de l'activité créatrice et inventive où en effet le geste produit non pas tautologiquement son propre sens mais $d u$ sens, c'està-dire qu'il tisse l'individu au monde ou aux collectifs dans lesquels il s'insère. De ce point de vue, Quand le geste fait sens est une contribution importante à l'analyse de l'articulation du sentir et du se mouvoir amorcée, il y a bientôt un siècle, par Erwin Straus : s'y esquissent les linéaments d'une méthode qu'on aimerait voir fleurir d'un examen philosophique des manières humaines d'être au monde par les gestes.

Romain Bigé

Bruno Frère \& Sébastien Laoureux (dir.), La Phénoménologie à l'épreuve des sciences humaines, Bruxelles, P.I.E. Lang, 2013, 171 pages. ISBN 9783035263138. Prix : 40,46€

Issu d'un colloque tenu à l'Université de Liège en mai 2007 sous le titre «Usages de méthodes phénoménologiques en sciences humaines », cet ouvrage collectif est une contribution importante à l'étude des rapports interdisciplinaires entre phénoménologie et sciences humaines. Bruno Frère et Sébastien Laoureux, coauteurs de l'introduction, résument à cet effet qu' « il s'agit en somme d'en faire ce qui n'avait pas encore été proposé explicitement : un outil d'analyse du monde et des choses, ici et maintenant » (p. 15). Les textes réunis s'inscrivent d'emblée dans une démarche préoccupée par le caractère «opératoire» (p. 9) des ressources mobilisées, leur usage étant restreint dès le départ par la référence à la réalité empirique. Il s'agit ainsi de mettre en évidence les innovations et les potentialités d'explicitation qu'elles permettent pour l'investigation empirique dans les sciences humaines, de l'anthropologie à la sociologie, en passant par la géographie et la psychanalyse. De façon générale, l'ouvrage tente de répondre à la question : en quoi une approche phénoménologique en sciences humaines permet-elle de comprendre mieux les réalités étudiées, de les décrire dans leur totalité concrète? 
La ligne directrice de l'ouvrage est bien commode pour le lecteur issu d'une de ses disciplines, puisqu'elle requiert des auteurs de détailler leur emploi de la phénoménologie. Une attention particulière est accordée à l'attrait précis que prend ce point de vue dans les recherches exposées, « dès lors qu'elles se trouvent confrontées à une problématique qui exige d'elles une prise sur le monde vécu » (p. 9). Le lecteur appréciera très certainement les clarifications judicieuses des emprunts à la phénoménologie et le contact aisé qu'elles peuvent amorcer sur ses concepts. L'illustration du propos par le recours à l'exemple concret, à des terrains d'enquête préalablement circonscrits, dans d'autres cas en passant par une historiographie ou une généalogie des enjeux entourant un objet, favorise une présentation méthodique s'ajoutant au souci général d'accessibilité pour le chercheur en sciences humaines.

L'articulation ici mise de l'avant, où la phénoménologie est abordée comme outil d'analyse empirique, se justifie de toute évidence par la spécificité du travail en sciences humaines, mais aussi par la demande des récentes méthodes de recherche. On assiste, depuis plusieurs années déjà, à l'émergence d'approches qui revisitent les modes de connaissance interprétatif et phénoménologique, en prenant pour mobile l'expérience vécue et l'action des sujets dans le monde social et quotidien. Il est ainsi devenu répandu de quitter les approches surplombantes au profit d'un retour au monde vécu quotidien des acteurs afin de mettre en évidence ses ressources de sens accumulées et disponibles, leur éveil et leur objectivation sans cesse renouvelée. Au fil de ce déplacement de la problématique à l'intérieur des sciences humaines, certains ont pu apercevoir dans la phénoménologie une méthode entrant en synergie avec leur posture épistémologique. Comme l'indiquent Frère et Laoureux, «[i]l convient de plonger dans les vécus humains qui se tissent de rapports immédiats au corps, aux animaux, aux choses et aux autres. La phénoménologie semble être la perspective la plus pertinente pour décortiquer dans toute leur finesse la matière de ces vécus et leur teneur généralisable » (p. 9).

Par-delà la diversité des ressources phénoménologiques mobilisées au fil des contributions, de même que la variété des objets et des problématiques auxquels elles sont mesurées, le lecteur remarquera que le geste de recherche empirique s'accompagne du maintien, dans une certaine mesure, d'un regard phénoménologique par l'explicitation du sens des réalités à travers un mouvement de dés-objectivation. De toute évidence, les chercheurs regroupés dans cet ouvrage ne se satisfont pas des approches prédominantes de leur champ disciplinaire, y apercevant des dimensions oubliées de l'expérience vécue appelant des approfondissements. Le point de vue phénoménologique 
oriente ainsi la recherche en lien avec les modalités de la participation des sujets au monde, c'est-à-dire avec le «sens » qu'ils confèrent à leurs expériences, leurs conduites et leurs actions constitutives des réalités étudiées. C'est d'ailleurs un des aboutissements remarquables de cet ouvrage collectif que d'illustrer en quoi la phénoménologie peut favoriser une attitude réfléchie et critique au sein des sciences humaines. L'impulsion de la recherche provient alors d'un travail de redécouverte, pour ainsi dire, des réalités concrètes du monde de la vie ou Lebenswelt, relancé par une mise en doute des théories et des idées préconçues sur un objet dans leur prétention à en identifier et à en déterminer en soi l'expérience.

Dans cette perspective, l'empreinte de la phénoménologie dans les sciences humaines ne saurait correspondre à la seule diffusion de motifs phénoménologiques (p. ex. une vague exigence de description fine ou de prise en compte de la perspective de l'acteur) ou à des rapprochements occasionnels (par exemple l'influence de la théorie des réalités multiples d'Alfred Schütz sur Les cadres de l'expérience d'Erving Goffman), ni non plus emprunter la voie d'un tournant paradigmatique. À l'inverse, l'ouvrage se situant d'emblée au foyer de sciences empiriques, une fondation philosophique du domaine d'objet de chacune des sciences humaines, dès lors abordée comme des ontologies régionales, est difficilement souhaitable puisqu'elle opérerait à sens unique. Toute la difficulté d'un projet interdisciplinaire est par conséquent d'arriver à une information réciproque de l'un et l'autre des champs de recherche concernés, à une participation commune à l'étude d'un même objet qui revivifie les regards scientifique et phénoménologique.

Afin de pousser plus avant cette articulation, nous proposons de prendre pour point de départ notre propre ancrage, la sociologie. Dans ce contexte, une phénoménologie constitutive de l'attitude naturelle œuvrant au sein d'une sociologie interprétative, telle que développée de façon notoire par Alfred Schütz, constituerait une proposition digne de considération. La contribution de Bruno Frère et de Sébastien Laoureux, intitulée « Promesses et impasses sociologiques de la phénoménologie», présente un examen critique de cette tentative visant à penser conjointement phénoménologie et sociologie. Avec sa thèse intitulée Der sinnhafte Aufbau der sozialen Welt: Eine Einleitung in die verstehende Soziologie, publiée en 1932, Schütz défend une posture scientifique qui cherche à renouer avec l'attitude naturelle en se penchant sur le monde quotidien et ses formations de sens complexes condensées dans des opérations de pensée sophistiquées (synthèses, typifications, généralisations, formalisations, idéalisations), des signes et des symboles diversifiés, de même que des habitudes et des dispositions oppor- 
tunes selon où l'on se trouve. Grâce à une méthode de typification et d'idéation revisitant la sociologie wébérienne, il est alors possible de reconstruire les réalités étudiées de manière à rendre compte des structures du sens subjectivement visé au sein d'un système de connaissance objective.

De cette refondation de la sociologie, c'est l'apport proprement épistémologique que retient la postérité, notamment le courant de l'ethnométhodologie, et qui fait l'objet d'une attention renouvelée avec le récent courant de la «sociologie pragmatique », comme l'illustrent les auteurs de façon exemplaire. En effet, tout le différend de sociologues tels que Luc Boltanski, Laurent Thévenot, Philippe Corcuff et Antoine Hennion avec Pierre Bourdieu porte sur la fracture dualiste qu'il alimente entre « connaissance de sens commun » et « connaissance scientifique ». Dans le sillage de Schütz, la sociologie pragmatique va alors insister sur le pouvoir d'agir des sujets sociaux et leur faculté de juger de leur conduite et de leur action selon la diversité et la complexité des contextes de sens des sociétés contemporaines. Comme le soulignent Frère et Laoureux, cette perspective suppose que «la réflexion théorique est aussi l'apanage des acteurs » (p. 141), qui prennent continuellement position, espèrent, doutent, redoutent, jugent, estiment, critiquent, cogitent, etc.

Pourtant, par-delà la seule question de la posture épistémologique, le projet schützien serait trop strictement philosophique pour être sociologiquement recevable, selon les auteurs. En effet, Schütz aborde le champ empirique de la sociologie comme une ontologie régionale à partir de la phénoménologie de Husserl pour en expliciter les structures invariantes et fondamentales. Cette approche de la sociologie, qui œuvre en-deçà du matériau empirique constitué de la Lebenswelt en reconduisant les descriptions eidétiques de Husserl obtenues dans la réduction phénoménologique, n'est pas sans poser un problème, puisqu'elle revient à « appliquer le transcendantal à l'empirique» (p. 142). Selon Frère et Laoureux, cette idée d'application traverse l'œuvre de Schütz jusqu'à son approche du social en première personne, qualifiée alors $\mathrm{d}^{\prime}$ '《impasse subjectiviste» (p. 145). Somme toute, on peut déplorer cette appréhension de la phénoménologie comme marquée d'avance par la réprobation, à défaut d'examiner la pertinence de l'expérience subjective et intersubjective du social pour la sociologie. Cela tend à escamoter les ajustements que les abords de l'objet, ici l'être-en-société, fournissent à la méthode phénoménologique, qui y puise un potentiel d'opérativité tout en se dépassant dans sa propre application, comme l'approche schützienne en est un cas exemplaire.

Il n'en demeure pas moins que le projet de refondation, comme l'ont bien vu les auteurs, comporte le risque de déboucher sur une relation

21 
formelle et statique aux champs empiriques, dans la mesure où les résultats de la description eidétique finissent par fonctionner comme des formes $a$ priori de l'objectivité qui demeurent étanches à la contingence du donné, à la dynamique d'ouverture du sens. Or, c'est précisément cette relation à l'objet qui se trouve mise en doute par la sociologie pragmatique, au profit d'une perméabilité des catégories sociologiques aux « rapports intentionnels que les gens ont avec le monde et les autres et la façon dont ils le soumettent (ou se soumettent mutuellement) à des épreuves, etc. » (p. 157). Il s'agit ainsi de tirer parti de l'heuristique inhérente aux subjectivités pour la genèse de catégorie. Les orientations, les attitudes vécues et les diverses prises de position par lesquelles les sujets s'engagent dans le monde constituent autant d'opérations sur le sens, qui participent à la catégorisation descriptive de l'objet et de ses dimensions contextuelles.

Cette ouverture potentielle du modèle conceptuel au nouveau et à l'aléatoire laisse envisager un autre rapport entre le transcendantal et l'empirique, à la faveur de l'empirique. Une «co-générativité » des deux plans, conformément au sens que lui donne Natalie Depraz dans ses travaux, amènerait l'analyse des champs empiriques à concrétiser et à affiner les catégories descriptives de l'expérience transcendantale qui, en retour, en réinvestirait les potentialités structurantes en y opérant des différenciations. On peut regretter que cette articulation plus franchement interactive et dynamique ne soit pas examinée en poursuivant le projet de Schütz, dans un ouvrage par ailleurs fort stimulant. Un des principaux enjeux de son article « Type and Eidos in Husserl's Late Philosophy » est justement de suspecter la prétention à dégager des invariants universels et transculturels de l'expérience du monde social, ou eidè. Insistant sur leur contingence au niveau culturel, voire même personnel, Schütz met l'accent sur les types, qui demeurent valables «jusqu'à nouvel ordre », non sans affecter l'identité des eidè, dès lors sujets aux vicissitudes, aux aventures et aux altérations de la vie empirique.

Simon Lafontaine 
Ernst Bloch, Études critiques sur Rickert et le problème de la théorie moderne de la connaissance, traduction, introduction et notes par Lucien Pelletier, Paris, Éditions de la Maison des Sciences de l'Homme, coll. «Philia », 2010, 470 pages. ISBN 9782735113040. Prix : $28 €$

Ernst Bloch n'est pas à proprement parler ce que l'on pourrait appeler un génie précoce. En effet, lorsqu'il qualifie son école d'«atroce» et «stupide » et qu'à ses enseignants il réserve l'adjectif de « racornis ${ }^{1}$ nous sommes normalement enclins à penser que l'on a affaire non pas tant à un rat de bibliothèque mais bien plutôt à un cancre. Or, entre cette période difficile de l'adolescence et L'Esprit de l'utopie - le premier ouvrage contenant déjà le souffle d'une œuvre systématique, paru quand son auteur avait 33 ans -, la découverte d'un chemin intellectuel et d'une forme d'approche de la vie académique et philosophique s'est produite. Jusqu'à présent, cette période de clivage qui va de l'adolescent rebelle au philosophe hardi - et dans laquelle se produit la véritable genèse de sa pensée - est restée dans l'ombre. La recherche récente doit sans doute à Lucien Pelletier l'effort de s'être obstiné à comprendre l'origine de certains concepts-clefs d'une œuvre encore assez méconnue dans le milieu francophone.

Les Études critiques sur Rickert et le problème de la théorie moderne de la connaissance sont le résultat bibliographique d'un tandem : il s'agit certes, principalement, de la thèse doctorale présentée par Ernst Bloch à la fin de ses études à l'université de Würzburg en 1908 mais, à côté de cela, il y a partout dans le livre la marque puissante du travail et de la personnalité du traducteur. Si le texte de Bloch n'occupe qu'un peu plus d'une centaine de pages, le vaste appareil critique avec lequel Pelletier l'enrichit, prend la plupart du volume. À travers une introduction et plus de 500 notes explicatives, le traducteur rend compréhensible le texte au lecteur actuel, en même temps qu'il nous livre sa propre interprétation de l'auteur.

Le livre ne se contente donc pas d'offrir aux lecteurs francophones l'intégralité du texte allemand, mais il va jusqu'à établir une version que l'on pourrait considérer comme canonique et ce, même par-delà les textes disponibles actuellement dans sa langue d'origine. Il semblerait que Bloch n'ait jamais eu de grande estime pour sa thèse doctorale. Celle-ci, par exemple, n'a pas été reprise dans la Gesamtausgabe sinon comme faisant partie, dans une version très abrégée, du volume complémentaire de l'œuvre

\footnotetext{
${ }^{1}$ Ernst Bloch, Traces (1959), tr. fr. par P. Quillet et H. Hildenbrand, Paris, Gallimard, 1968, p. 57 et p. 59.
} 
complète appelé Tendenz, Latenz, Utopie ${ }^{1}$. Le seul texte complet de la thèse dont le chercheur, sans faire recours à un travail d'archive, dispose désormais est donc bien celui de l'édition française. Celle-ci devient ainsi une trahison en quelque sorte de la volonté de Bloch afin de retracer, felix culpa, le noyau matriciel des concepts les plus originels de son auteur, ceux-là mêmes qui lui ont valu une place d'honneur dans l'histoire de la philosophie.

Le volume s'ouvre avec une introduction générale du livre, écrite par Lucien Pelletier, qui semblerait d'ailleurs avoir pour but le fait de rendre futile tout compte-rendu postérieur du livre, tant elle aspire à l'exhaustivité qui lui est permise. Y sont clairement indiqués le besoin de prendre au sérieux l'apport de Bloch à la philosophie, les circonstances biographiques dans lesquelles la thèse traduite a été écrite, ainsi que le contexte intellectuel de son époque peint à grands traits. On y trouve aussi un résumé des idées principales du texte de Bloch et des possibles avenues de recherche qui pourraient s'ouvrir à partir de la redécouverte des œuvres marquantes de la génération des penseurs qui ont servi à Bloch d'interlocuteurs ou qui sont issus du même contexte socio-culturel.

Vers la page 50 c'est Bloch qui prend finalement la relève. Du texte en général, ce qui surprend le plus est le fait que le lecteur avisé de Bloch découvre déjà dans la thèse écrite à 23 ans des intuitions fortes, des concepts centraux, de nets penchants qui seront développés dans l'œuvre postérieure dont la continuité et la cohérence, à la lumière du texte de 1908, est étonnante. Mais, d'un autre côté, on sera surpris par un certain manque de rigueur par rapport à la forme académique de la philosophie, du moins telle qu'elle se présente actuellement. Il en résulte que des pages entières peuvent être tout à fait incompréhensibles pour le lecteur qui lit le texte cent ans après sa parution, et qui a alors perdu le Sitz im leben du texte original.

La thèse s'articule autour de trois parties, clairement différenciées dans le texte de Pelletier mais pas dans l'original allemand, où celles-ci ne sont distinguables que sur la table des matières: d'abord, le jeune philosophe dresse le bilan de la philosophie que sa génération reçoit et entend déjà bien son programme, entre autres, comme une lutte contre le positivisme, compris ici lato sensu. À travers une critique de Lamprecht, Mach, James et Riehl, Bloch perçoit le positivisme comme une attitude épistémologique et une façon de s'approcher de l'histoire. Au diapason des nouvelles inventions de la révolution industrielle, à la génération postromantique il fut imposé la tâche de se réapproprier d'une façon nouvelle du donné autant naturel qu'historique. Pour Bloch le positivisme est donc la conséquence de cette

${ }^{1}$ Ernst Bloch, Tendenz, Latenz, Utopie, Frankfurt, Suhrkamp, 1978, p. 55-107. 
rencontre. Le résultat a été un monde dissout épistémologiquement en un conglomérat de sensations diverses de nature spatiale ou chimique, capables d'expliquer, sans mystères, les plus fines activités de l'esprit telles que l'amour ou l'héroïsme, dorénavant compréhensibles dans leur fonctionnement comme peuvent par exemple être compréhensibles les chaudières des machines à vapeur. La science historique a quant à elle vu naître de nouvelles approches pour lesquelles la dimension socio-économique et surtout l'histoire culturelle ont commencé à être pris en compte, quoiqu'encore en faisant recours à une causalité historique linéaire, issue des sciences de la nature.

La seconde partie de la thèse, la plus longue d'ailleurs, s'occupe de dresser un bilan sur la pensée de Rickert, dont la philosophie n'arrive pas complètement, selon Bloch, à se défaire du positivisme. Pour Rickert, la réalité consiste en « un matériau absolument incommensurable de faits, qui se donne en une infinité tout autant intensive qu'extensive» (p. 68). Mais, pour percer l'irrationalité du donné, deux options sont possibles : du point de vue du général, l'instrument dont nous disposons pour y avoir accès seraient les sciences de la nature; du point de vue du particulier, par contre, cette responsabilité revient à la science historique. Or, si sujet et nature sont de la même étoffe, rétorque Bloch, l'irrationalité de la nature serait aussi extensible au sujet. Il est par conséquent contradictoire de prétendre que le donné irrationnel soit cognitivement accessible à un sujet qui garantirait sa validité alors que ce sujet partage l'irratio du donné même. Si ce qui définit l'irrationnel c'est le fait d'être fugace et incapable de régularité, il est absurde de prétendre que celui-ci puisse être circonscrit dans des lois. Pour que la connaissance soit possible, il faudrait plutôt d'après Bloch postuler qu'il y ait des zones de la réalité qui sont en lutte pour venir à bout de leur propre irrationalité et atteindre le seuil de la logique. Seule cette impulsion de l'irrationnel vers la connaissance peut expliquer le surgissement de la conscience.

On comprend mal comment le donné, incommensurable, impénétrable et non déductible, peut être subsumé en concepts et être l'objet d'un questionnement de notre part. Ce chemin problématique qui va du fait au concept s'exprime de façon paradigmatique lorsqu'à partir de certains phénomènes, la science veut établir des lois qui permettraient d'en prédire d'autres. Or le prévoir de la science ne peut jamais subodorer des changements inattendus mais à peine découvrir déductivement une régularité future.

Si l'antérieur pose déjà un problème aux sciences de la nature, il en va de même pour le savoir historique puisqu'il poursuit une saisie de l'individuel tout en excluant le côté intérieur, voire psychologique des 
événements. Face à cela, Bloch verra chez Lamprecht ou W. Wundt l'effort de revendiquer la nécessité d'un usage de la psychologie dans l'histoire, notamment pour arriver à une saisie de l'esprit d'une époque ou pour comprendre des comportements apparaissant comme typiques de certains moments de l'histoire, alors qu'ils sembleraient déplacés ou tout simplement incompréhensibles dans d'autres moments. Chez Simmel, par exemple, les phénomènes de masses sont d'une importance majeure dans la réflexion.

Mais en niant, à l'instar de Rickert, la possibilité de se servir d'une psychologie de la culture dans la compréhension des faits historiques on bloque le chemin qui permettrait de comprendre par exemple le fait paradoxal de certaines individualités du passé qui semblent avoir vécus des choses non contemporaines, qui excédaient l'esprit de leur époque. Pour Bloch, par contre, l'action des sujets introduit de l'inédit dans l'histoire, une sorte de saut entendu comme intervention de la liberté et donc comme une introduction du transcendant dans le temps.

Dans cette partie centrale de la thèse, apparaissent déjà des conceptsclefs de l'œuvre de Bloch toute entière. C'est le cas par exemple des thèmes de l'utopie, de la réaction contre l'idéologie du progrès, de l'idée de noncontemporanéité ou de la notion d'obscurité de l'instant vécu. Pour bien cerner ces motifs, l'appareil critique avec lequel Lucien Pelletier agrémente le texte original s'avère d'une importance capitale. Il a le mérite de créer une sorte d'illusion d'optique chez le lecteur. La thèse doctorale du jeune Bloch apparaît, à notre avis, comme une sorte de cathédrale toute construite avec ses autels et ses ex-voto complets. Certes, Pelletier arrive à démontrer comment la plupart des thématiques de l'œuvre postérieure du philosophe sont déjà, soit explicites ou mûres, soit in nuce dans le texte de 1908 quoique dans une forme que semblerait parfois trop consciente pour la pensée du doctorant Ernst Bloch. Une chose est vraie, les Études critiques sur Rickert contiennent sans doute le pathos de la jeunesse du philosophe d'une façon encore plus forte que L'Esprit de l'utopie. Pourtant, il ne faudrait pas oublier que toutes les impulsions juvéniles trouvent une réelle satisfaction postérieure. Si la pensée la plus originale de Bloch est celle de sa jeunesse, ce ne sont pas les simples intuitions du jeune homme ce que peut retenir de lui l'histoire de la philosophie, mais plutôt les grandes formulations de la maturité. Pour cela même, l'étude historico-philologique d'immense valeur que Pelletier a réalisée reste heureusement un chemin parmi l'éventail d'approches possibles pour l'étude de cette œuvre.

La troisième partie du texte de Bloch semble oublier presque complètement l'analyse de l'œuvre de Rickert et essaie de chercher chez des auteurs de son époque ou récents le souffle d'une nouvelle forme de 
métaphysique. En tant que témoins du moment philosophique de la fin du siècle et du début d'un autre, ainsi que de la quête d'une nouvelle expression philosophique qui aille au-delà du néokantisme et du positivisme duquel Rickert n'est pas parvenu à se défaire, Husserl, Meinong, Scheler, Eucken, Tönnies et Cohen sont convoqués.

Selon Bloch, ce qui chez Husserl est novateur et révolutionnaire consiste dans le fait d'avoir libéré le donné du carcan de l'irrationalité dans lequel il est resté enfermé chez Rickert. En effet, pour Husserl le début des pensées se trouve dans un ordre originaire qui relève du pré-psychologique. De Meinong, Bloch retient l'importance de son étude sur les assomptions. De Max Scheler il est repris une bonne partie des critiques adressées à la méthode transcendantale kantienne, notamment ce qui concerne l'espace et le temps. Chez Eucken, Bloch problématise la relation sujet-objet comme ce qui constituerait le propre de la vérité. De Tönnies intéressent Bloch les relations étudiées entre communauté et société. De Cohen par-delà son apriorisme est valorisée l'idée d'une origine infinitésimale du quelque chose comme point archimédien de la compréhension du réel.

Le volume s'achève enfin avec les notes du traducteur, qui sont plus que de simples éclaircissements sur des points obscurs, et constituent un vrai commentaire et une aide précieuse à la lecture. En Virgile, l'intention de Pelletier est de simplement aider le lecteur de Bloch «à s'approcher le plus possible des sources de la philosophie blochienne et à en cerner les idées originelles en les situant dans leur contexte " (Lucien Pelletier, "Introduction », p. 13). Or le résultat semble aller bien au-delà de ce modeste objectif. En ce sens, la clarté du traducteur non seulement brille sur l'opacité de la langue blochienne, mais parvient même, à sa façon, à esquisser le tableau du panorama philosophique d'une période de la philosophie allemande encore assez peu étudiée et peu traduite en français. Au-delà de cette tension absurde entre le simple commentateur et l'auteur essayiste, Lucien Pelletier est un des plus fins historiens des idées de langue française. À partir de Bloch, il arrive à dessiner tout un tableau de la philosophie allemande de la fin du XIX ${ }^{\mathrm{e}}$ siècle et du début du $\mathrm{XX}^{\mathrm{e}}$ qui se complète avec le reste de sa production bibliographique récente ${ }^{1}$.

Aníbal Pineda Canabal

\footnotetext{
${ }^{11}$ Il vaut certainement la peine de mentionner ici son article « Pourquoi Bloch a-t-il fait sa thèse sur Rickert? », in Francesca Vidal (éd.), Bloch Jahrbuch 2011, Utopien von Zivilgesellschaft, Mössigen, Talheimer, p. 142-170).
} 
AA.VV., L'Archi-politique de Gérard Granel, Mauvezin, Trans-EuropRepress, 2013, 402 pages. ISBN 9782905670595 . Prix : $32 €$

Les vingt-quatre essais qui composent ce volume, issus d'un colloque éponyme tenu au Centre Culturel International de Cerisy-La-Salle en juillet 2012, constituent à n'en pas douter un témoignage exemplaire de l'actualité et de la fécondité de la pensée de Gérard Granel. Complétant les deux substantiels recueils déjà consacrés au philosophe ${ }^{1}$, le présent ouvrage se concentre plus précisément sur ce qui, selon l'expression granélienne, est décisif, à savoir le politique ${ }^{2}$. Pour Granel, en effet, la philosophie ne peut aujourd'hui exister légitimement qu'à la condition de sa conversion en une « archi-politique », dont la mission historiale consiste à saisir l'archè du politique, cette décision originelle, prise au sein d'une forme de vie, qui institue le politique (p. 239). Cette tâche devient d'autant plus pressante qu'à l'heure où «plus rien ne résiste au Capital dès lors que la production dont il est le terminus a quo et le terminus ad quem se paye de l'ablation du politique, et par conséquent de l'éthique $»^{3}$, la philosophie, de même que toute interrogation conceptuelle fondamentale, est sommée de procéder selon la logique productiviste qui définit le Capital (p. 195 et p. 63). S'associant à cette entreprise de renouvellement de la philosophie, les essais ici publiés sont autant de gestes de résistance à cette «mise au pas » que constitue la transformation « de tout étant en une marchandise échangeable » (p. 197).

Dans un remarquable article, Andrea Cavazzini revient sur les étapes essentielles de la «réinscription » de la pensée marxienne développée par Granel, qui fut marquée par la tentative de dégager « l'opération par laquelle Marx érige en concept ontologique la donnée historico-sociale de la production capitaliste-industrielle » (p. 57). Ainsi que le note à juste titre Cavazzini, cette revalorisation du référentiel de la production, qui désigne le phénomène qui manifeste l'essence de notre monde $^{4}$, a pour enjeu de « laisser percer ce qui n'a jamais percé dans l'histoire, à savoir le travail en tant qu'il n'est pas déjà et d'avance détourné, canalisé et organisé comme

\footnotetext{
1 Jean-Luc Nancy \& Élisabeth RIGAL (éd.), Granel, l'éclat, le combat, l'ouvert, Paris, Belin, 2001 et Éric Clémens et alii, Gérard Granel ou la rigueur du dénuement, Mauvezin, T.E.R., 2012.

${ }^{2}$ Gérard Granel, Écrits logiques et politiques, Paris, Galilée, 1990, p. 55.

${ }^{3}$ Ibid., p. 51.

${ }^{4}$ Gérard Granel, Apolis, Mauvezin, T.E.R., 2009, p. 55.
} 
simple force de travail $»^{1}$. La critique de la production nous permet d'accéder à une nouvelle conception du travail. Comment cependant laisser percer, du sein de la production capitaliste, ce dont nous n'avons jamais fait l'expérience?

C'est à la question de savoir comment le travail peut à la fois être ce qui nous ouvre au monde et ce qui nous en prive que Frank Fischbach consacre son essai. Comme le rappelle celui-ci à la suite de Granel lisant Heidegger, l'être-au-monde est essentiellement être-à-la-tâche : c'est grâce au travail que l'homme accède à la dimension même du monde, qu'il s'ouvre à une historicité qui lui est propre (p. 154). Or, sous l'emprise de la production capitaliste, le travail ne permet plus de transformer le temps en un temps historique : ce qui est produit, ainsi que Marx l'indiquait dans Le Capital, apparaît comme une collection, un amas, ce qui par conséquent ne peut jamais constituer un monde (p. 158) ${ }^{2}$. Ce n'est donc pas le travail qui domine notre époque, mais le travail universellement productif. D'après Fischbach, l'échappatoire ne consiste pas à libérer le travail de l'emprise de la forme-Capital, de telle façon qu'un « bon vieux réel du travail », opprimé et dominé, réapparaisse à cette occasion - ce que, d'après lui, suggérerait Granel. Alors que sous le règne du Capital, le travail, selon Fischbach, devient difforme, sa libération signifie son élévation au rang de forme, dont l'unification se réaliserait de façon immanente au travail lui-même.

S'il est vrai que Granel semble effleurer l'idée que le travail aurait encore à percer et que, d'un autre côté, il paraît identifier parfois la production capitaliste et le travail en général, il ne faut pas considérer la première affirmation au pied de la lettre, comme le fait Fischbach, précisément pour la raison que, selon ce qu'induit la deuxième proposition, Granel a bien vu que le travail possédait toujours une « face obscure ». Il ne sert à rien de jouer la forme-Capital contre la forme-Travail, ce qui serait revenir à une sorte d'essentialisme métaphysique, à plus forte raison quand

1 Gérard Granel, «Cours sur Gramsci, 1973-4», p. 223, disponible sur www.gerardgranel.com

${ }^{2}$ Ainsi que l'indique Pierre Zaoui : «Rigoureusement, on ne peut pas dire que pour Marx le monde capitaliste moderne "est" une immense accumulation de marchandises. Il nous apparaît ainsi, mais c'est une illusion, l'illusion de la prospérité qui a justement aveuglé pour Marx et les Anciens et les économistes bourgeois qui n'ont pas pu, et puis pas su, voir que sous cet apparent rapport entre des choses se cachaient des rapports entre les hommes parfaitement déterminés les rapports de travail ou rapports de production » (p. 395).

29

Bull. anal. phén. XIV 4 (2018)

https://popups.uliege.be/1782-2041/ @ 2018 ULiège BAP 
celui-ci, selon la variante romantique proposée par Fischbach (p. 161) ${ }^{1}$, s'appuie sur l'idée que « nous » posséderions parfois le souvenir d'un travail authentique, quand il permettait encore l'accès à un monde. Ce n'est pas uniquement le travail en régime capitaliste qui dépossède l'homme d'une histoire propre ; arracher le travail au régime de la production n'est pas suffisant pour écarter le spectre de l'aliénation - et, à vrai dire, il faut espérer que la fuite hors de la forme-Capital n'entraîne pas l'advenue de la « figure du Travailleur » comme, après Jünger, Fischabch semble l'appeler de ses vœux. Il s'agit de conjurer ce qui, au sein du travail lui-même, produit l'aliénation. Comme Granel l'a très bien perçu, si l'accès au monde est le fait du travail, il est aussi ce qui, sous quelque forme que ce soit, tend à s'autoreproduire et à s'auto-accroître, c'est-à-dire à produire un monde que nous expérimentons bel et bien, et non pas seulement de façon négative, sans que nous ne puissions jamais vraiment réussir à nous en extraire, à nous en détacher. La production n'est ni une perversion du travail, ni une rupture avec celui-ci ; c'est bien plutôt le travail qui semble toujours déjà receler en lui la possibilité de la production. Si c'est incontestablement à partir du travail que l'on accède au monde, l'éventualité que celui-ci n'en soit pas vraiment un, qu'il soit juste « un immense entassement de marchandises », est toujours une disponibilité présente au cœur même du travail. D'ailleurs, Heidegger n'affirmait-il pas, dans Sein und Zeit, que la quotidienneté médiocre du Dasein n'est pas un simple aspect, mais constitue a priori une structure de l'existentialité, ce qui ne peut être dépassé ?

La critique de la subjectivité initiée par Granel ne peut en aucun cas déboucher sur l'idée qu'il existerait quelque chose comme un travail naturellement créateur et favorisant le libre accomplissement de soi, que la production aurait perverti, mais auquel il serait possible, suivant une conception romantique, de faire retour. L'idée que le travail serait originellement comme tel repose sur une conception de la subjectivité qui, comme le note Pierre Zaoui dans un très beau texte, constitue « une adaptation cynique aux exigences de reproduction de la "grande forme" du marché capitaliste mondial» (p. 388), une «grande forme» qui, comme le dit Granel, «a fabriqué le "sujet", comme sujet rationnel et sujet productif, comme sujet politique et comme sujet littéraire, comme sujet psychologique et comme sujet créateur $»^{2}$. Se distancier de ces deux conceptions du travail (ou bien comme aliénation ou bien comme accomplissement) revient à élaborer une

\footnotetext{
${ }^{1}$ À cet égard, on peut lire la mise au point d'Élisabeth Rigal dans «L'inflexion wittgensteinienne », p. 287.

${ }^{2}$ Gérard Granel, Écrits logiques et politiques, op. cit., p. 328.
} 
nouvelle conception de la subjectivité - à chaque conception du travail correspond une conception de la subjectivité - , c'est-à-dire de la temporalité, qui, tout en affirmant toujours le caractère existential du travail, en assumerait les aspects contradictoires. Pour atteindre ce but, il nous faut procéder, comme Granel nous y invite, à une lecture croisée de Hume et de Heidegger.

Alors que la subjectivité capitaliste est corrélée à une expérience de la temporalité conçue telle une suite ou une quantité d'instants, dont la tonalité fondamentale semble effectivement être l'angoisse - une tonalité qui nous révèle notre impossibilité de participer à une histoire commune -, une expérience de la temporalité qui prétendrait se soustraire à la logique productiviste doit lui opposer le temps plein et fini d'une tâche qui soit à ellemême sa propre fin. Au travail qui tend à s'auto-reproduire, il faut objecter le temps long d'un travail qui puisse devenir jouissance ou plaisir ${ }^{1}$, sans jamais que ce temps du plaisir puisse être mesuré : «Aux époques où l'industrie et les arts sont florissants, les hommes sont pris dans une occupation perpétuelle, et la récompense qu'ils goûtent, autant que les plaisirs qui sont les fruits de leur labeur, est cette occupation elle-même $»^{2}$. Le plaisir devient cette autre tonalité fondamentale qui, opposée à celle de l'angoisse, nous ouvre non pas à la liberté pour la mort, comme possibilité la plus propre, mais au renvoi de la mort comme ce qui n'adviendra peut-être jamais, ou alors seulement comme ce qui passe.

Aurélien Zincq

Federico Boccaccini (dir.), Lotze et son héritage. Son influence et son impact sur la philosophie $d u \quad X X^{e}$ siècle, Bruxelles, P.I.E. Peter Lang, coll. «Philosophie \& Politique», 2015, 274 pages. ISBN 9782875742780. Prix : $48,20 €$.

Le présent ouvrage constitue la première monographie en français consacrée au philosophe allemand Rudolf Hermann Lotze. Se focalisant sur son « legs » à la philosophie allemande et américaine, il offre un témoignage

${ }^{1}$ Ibid., p. 316 sq. Cf. David Hume, «Du raffinement dans les arts », dans Essais moraux, politiques et littéraires, trad. fr. G. Robel, Paris, P.U.F., 2001, p. 444-445, cité par Xavier Papaïs, p. 279.

${ }^{2}$ Gérard Granel, Écrits logiques et politiques, op. cit., p. 316.

31

Bull. anal. phén. XIV 4 (2018)

https://popups.uliege.be/1782-2041/ @ 2018 ULiège BAP 
de premier plan de l'influence profonde, bien que parfois discrète comme c'est le cas dans la réception francophone, de la pensée d'un auteur-charnière dans le développement de l'histoire de la philosophie aux XIX et $\mathrm{XX}^{\mathrm{e}}$ siècles. Lotze est en effet une figure centrale pour qui veut comprendre l'apparition et la transformation de ces courants majeurs que sont la philosophie scientifique, le néokantisme dans ses diverses formes, la phénoménologie, le pluralisme jamesien ou encore le réalisme de Santayana. Héritier de l'ancienne Chaire de Herbart à Göttingen (1844-1880) et prédécesseur de Dilthey à l'Université de Berlin (1880-1881), Lotze fut également, avec Franz Brentano le maitre de Carl Stumpf (lequel soutint sa dissertation sur Platon et sa thèse d'habilitation sous sa direction), le professeur de Wilhelm Windelband et de Gottlob Frege. Bien qu'il n'ait pas fondé d'école (sans doute, comme l'explique Stumpf, à cause d'une absence d'unité doctrinaire), ses écrits et ses thèses philosophiques ont connu un retentissement remarquable, que ce soit dans la philosophie de langue allemande, que dans les mondes francophone et anglophone.

En témoignent, sur ce dernier point, les traductions rapides, en français et en anglais, de ses principaux ouvrages et articles. Dans le cas du français, dès 1881 la Medizinische Psychologie est traduite, rapidement suivie par la Metaphysik en 1883. Comme le signale F. Boccaccini (p. 12-13), Lotze sera commenté très vite par Ernst Reinhardt, Théodule Ribot, Charles Renouvier ou encore le jeune Jean Wahl dans Les Philosophies pluralistes d'Angleterre et d'Amérique, en 1920. Mais sa postérité dans le monde francophone s'arrêtera à ce point. Du côté anglophone, la réception fut, ainsi qu'on le sait déjà, plus importante et prolifique. Outre la Logik qui fut traduite de bonne heure, la Metaphysik, Mikrokosmus et des notes de cours de Lotze sur différentes matières (six volumes publiés entre 1884-1887) permettront à nombre de philosophes, théologiens et scientifiques de première importance de prendre connaissance des travaux du philosophe allemand. Parmi les contributions du volume, celle de Stefano Poggi et Michele Vagnetti est consacrée à une analyse minutieuse d'un certain nombre d'idées de Lotze qui eurent une influence, si ce n'est décisive du moins appréciable, sur le développement de la philosophie de William James. Est ainsi apprécié le rôle de Lotze dans la formation de l'un des philosophes les plus influents de la fin du XIX siècle. Federico Boccaccini, quant à lui, étudie en profondeur la connexion entre la philosophie scientifique allemande postkantienne et le réalisme américain, en particulier chez George Santayana. La thèse de l'auteur, originale, est que le kantisme de Lotze offrit « une version acceptable de scientisme capable de s'accorder avec la foi religieuse » (p. 171). Lotze apparaît dès lors non seulement comme un maillon indispensable pour 
comprendre la réception du kantisme aux U.S.A., mais également comme l'inspirateur d'un style de pensée réaliste, critique et foncièrement ouvert aux apports des science de la nature qui fera florès outre-Atlantique (et auquel Santayana permettra précisément de donner une certaine amplitude).

Concernant ses principales thèses philosophiques, deux sont sans doute à retenir en priorité : sa théorie de la validité (Geltung) et sa théorie des signes locaux. On notera également que l'importance qu'il accorde aux sciences naturelles (tout en professant un anti-naturalisme) auront une influence majeure sur le développement de la philosophie scientifique dans la seconde moitié du XIX ${ }^{\mathrm{e}}$ siècle.

Parmi les nombreux courants que Lotze influença, c'est sans doute la phénoménologie qui lui est le plus redevable. Dans le riche article qu'il consacre à la théorie des signes locaux et à la controverse empirismenativisme dans la perception de l'espace, Denis Fisette n'hésite d'ailleurs pas à considérer Lotze, grâce à la place qu'il a occupée dans ce débat, comme l'un des prodromes de la méthode phénoménologique, comprise comme approche radicalement descriptiviste des phénomènes sensibles. Dans ce jeu d'influence, Denis Seron entrevoit, à partir d'une analyse minutieuse du projet lotzéen de psychologie physiologique, une réelle proximité entre les positions de Lotze à propos de l'analyse de l'esprit et la psychologie de Brentano «mais aussi, plus largement, quelque chose qui est à proprement parler une phénoménologie » (p. 41). En effet, comme il le souligne, le questionnement de Lotze sur l'esprit n'est pas d'abord un questionnement métaphysique sur la «substance » psychique, mais une méthode d'investigation privilégiant la seule description des phénomènes psychiques. Bien sûr, Brentano critiquera Lotze, dans la Psychologie vom empirischen Standpunkt, pour ne pas s'être débarrassé du concept d'âme ni ainsi affranchi de toutes présuppositions métaphysiques, mais cela empêche-t-il véritablement Lotze, dès lors qu'il fait œuvre de psychologue, de s'en tenir aux principes d'une «psychologie sans âme»? On peut en douter.

Deux articles sont dédiés à l'importante théorie lotzéenne de la validité. Pour rester dans la veine phénoménologique, l'article de Maria Gyemant entreprend de montrer l'étendue réelle de l'influence exercée par Lotze sur le jeune Husserl eut égard à la question de l'idéalité. Contre les vues généralement admises quant au rapport de Husserl à Lotze à propos de la théorie des Idées, et grâce à la consultation d'un manuscrit de Husserl non publié (K I 59), l'auteure réussit à démontrer que la théorie de l'idéalité élaborée dans les Logische Untersuchungen est en réalité tout à fait originale et ne doit que très peu à l'héritage lotzéen. Au contraire, argumente Maria Gyemant, elle a constitué pour Husserl une première occasion d'élaboration 
d'une théorie de l'idéalité précisément en prenant distance par rapport à la conception lotzéenne de l'objectivité des Vérités. Malgré ce contrecoup porté à ce que l'on avait depuis longtemps admis, dans la littérature phénoménologique, comme l'un des points-clés de l'importance, voire du génie de Lotze concernant la question de la validité, Arnaud Dewalque va tenter de réévaluer le sens et la portée de la théorie lotzéenne de la validité en tâchant de mettre en évidence comment elle est en étroite connexion avec ce que, dans le langage de Husserl, on peut appeler une analyse éidétique des phénomènes sensibles (p. 74). En d'autres termes, le «monde du représentable » (Welt des Vorstellbaren) est soumis à des légalités éidétiques. Plus fort encore, la notion de validité telle que développée par Lotze «ne prend son sens, pour l'auteur, qu'en référence à un monde sensible possédant une structure phénoménale et objective susceptible d'être décrite» (p. 75). Dans un cadre esthétique, $\mathrm{C}$. Morel va développer une réflexion sur la théorie lotzéenne de la validité et sa récupération par les néo-kantismes, en particulier celui de l'école de Bade-Wurtemberg.

L'article de Jocelyn Benoist constitue sans doute la proposition exégétique la plus audacieuse du volume. Prenant sa source dans les débats contemporains (ou qui nous furent contemporains il y a quelques années) sur le rapport du conceptuel et du perceptuel, l'auteur entreprend de mettre en lumière la façon dont la théorie lotzéenne des concepts sériels, exposée par le philosophe allemand dans sa Logik, permet d'y offrir une contribution de premier plan. La thèse de l'auteur est que Lotze réussit à conserver la distinction entre le registre de la simple expérience perceptive et la capacité à thématiser explicitement une identité, tout en transformant la conception traditionnelle du concept comme généralité pour lui offrir plus de plasticité - et, ainsi, lui permettre de saisir le donné dans sa singularité. Le concept, pour Lotze, ne cherche pas à capturer dans le donné une marque distinctive ; il fonctionne bien plutôt de façon sérielle, traversant les singularités et les mettant en série (p. 156). Ainsi, par exemple, ce qui constitue l'être-bleu n'est pas que l'on puisse isoler, à travers une série de couleurs vues, l'élément précis qui serait cet être-bleu, mais plus simplement la «ressemblance de famille », la « modulation » ou encore la « configuration variable » qui nous fait percevoir une certaine couleur comme relevant effectivement de ce que l'on entend par l'être-bleu.

La série de contributions se prolonge par trois textes de Lotze, dont deux traductions inédites. Le premier texte reprend un article paru dans la Revue philosophique de la France et de l'étranger en 1877 et consacré à «La théorie des signes locaux ». Le deuxième texte est un extrait de la Logik, le chapitre précisément consacré à «La délimitation des concepts ». Enfin, 
« Sur le concept de beauté » donne une idée de l'esthétique de Lotze, encore trop peu étudiée. On soulignera le choix judicieux de l'éditeur du volume d'avoir choisi ces trois textes en traduction; tandis le deuxième est directement commenté par J. Benoist et par A. Dewalque, le premier l'est par Fisette et Seron et permet de comprendre un point de vue fondamental sur la controverse empirisme-nativisme ; le texte sur le beau, quant à lui, présente une facette de la production de Lotze malheureusement encore peu connue.

On ne peut que saluer la parution du volume dirigé par Federico Boccaccini, qui vient combler une lacune dans l'histoire de la philosophie de langue allemande du $\mathrm{XIX}^{\mathrm{e}}$ siècle. On ne peut que souhaiter qu'il suscite d'autres travaux sérieux sur un auteur dont il reste encore beaucoup à apprendre.

Aurélien Zincq 